\title{
Control del agua de lastre como fuente de ingreso de especies exóticas invasoras en puertos argentinos de la Cuenca del Plata
}

Control of ballast water as a source of invasive exotic species in Argentine ports of the River Plate Basin

Controle da água de lastro como fonte de entrada de espécies exóticas invasoras nos portos argentinos da Bacia do Prata

Mariana Inés Abelando ${ }^{1,2}$ https://orcid.org/0000-0003-4112-714X

*Autor de contacto: mariana.ines.abelando@gmail.com

${ }^{1}$ Prefectura Naval Argentina, Dirección de Protección Ambiental. Buenos Aires, Argentina.

${ }^{2}$ Instituto Universitario de Seguridad Marítima. Buenos Aires, Argentina.

Recepción: 24 Agosto 2020 Aprobación: 21 Octubre 2021

Esta obra está bajo una Licencia Creative Commons Atribución NoComercial 4.0 Internacional. 
Resumen: Gracias al avance tecnológico, la frecuencia de los viajes transoceánicos ha aumentado; y de manera involuntaria, también el transporte de especies de una región a otra, quebrantando barreras naturales. Cuando estas especies se convierten en invasoras, suelen generar graves impactos en la biodiversidad, la economía y la salud pública. Este estudio analiza la gestión del agua de lastre en los puertos argentinos situados en la Cuenca del Plata, basándose en datos publicados e inéditos acerca del cumplimiento de la normativa nacional e internacional, análisis de riesgo, tendencias y experiencias en otros países de la región. Luego de casi una década de trabajo, Argentina ha logrado un cumplimiento efectivo de la normativa vigente por parte de la flota mercante, lo que se ha visto reflejado en la ausencia de nuevas especies invasoras en la región. Los esfuerzos futuros para reducir los impactos de la invasión deben considerar la optimización de recursos e incorporar nuevas metodologías en el control del agua de lastre, particularmente la Regla D-2, así como también considerar las bioincrustaciones como otro vector de importancia.

Palabras clave: Argentina, bioinvasiones, BWM 04, control, salinidad.

Abstract: Thanks to the technological advances, the frequency of transoceanic voyages has increased and, in an involuntary way, the transport of species from one region to another, breaking natural barriers. When these species become invasive, they usually have serious impacts on biodiversity, the economy and public health. This study analyzes the ballast water management in Argentine ports located in the Del Plata Basin, based on published and unpublished data on compliance with the national and international regulations, risk analysis, trends and experiences in other countries of the region. After almost a decade, Argentina has achieved an effective compliance of the rules in force by its merchant fleet, what has been reflected in the absence of new invasive species in the region. Future efforts to reduce the impacts of invasions should consider the optimization of resources and the incorporation of new technologies for ballast water control, particularly Regulation D-2, and they should also consider biofouling as another vector of importance.

Keywords: Argentina, bioinvasions, BWM 04, control, salinity.

Resumo: Graças ao avanço tecnológico, a frequência das viagens transoceânicas aumentado e, involuntariamente o transporte de espécies de uma região a outra, quebrantando barreiras naturais. Quando essas espécies tornam-se invasoras, muitas vezes têm sérios impactos sobre a biodiversidade, a economia e a saúde pública. Este estude analisa na gestão da água de lastro nos portos argentinos situados na Bacia do Prata, com base em dados publicados e dados inéditos acerca do cumprimento das regulamentações nacionais e internacionais, a análise do risco, as tendências e expêriencias em outros países da região. Depois de quase uma década de trabalho, a Argentina tem assegurado o cumprimento efetivo das regulamentações vigentes pela frota mercante, o que foi refletido na ausência de novas espécies invasoras na região. Os esforços futuros para reduzir os impactos da invasão devem considerar a optimização de recursos e incorporar novas metodologias ao controle de água de lastro, particularmente a Regra D-2, assim como devem considerar as bioincrustações como outro vetor de importância. 
Palavras-chave: Argentina, bioinvasões, BWM 04, controle, salinidade.

\section{INTRODUCCIÓN}

Las especies nativas o autóctonas comprenden desde microorganismos hasta plantas y animales superiores; son características de cada ecosistema y rara vez suelen traspasar sus límites naturales de distribución, siendo las corrientes oceánicas, las aves y los peces quienes contribuyen en su dispersión natural. A lo largo de los años, los seres humanos han estado movilizando organismos de un lugar a otro del planeta, atravesando océanos y quebrantando barreras biogeográficas naturales (Schwindt, et al., 2018). Muchas de estas especies han sido capaces de establecer nuevas poblaciones fuera de sus límites naturales y amenazar a las especies nativas, causar daños ecológicos y hasta convertirse en una amenaza para la salud y la economía de las poblaciones, por lo que se catalogan bajo el término de invasoras (Carlton, 1996, Pimentel, et al., 2005). Estas incursiones han mostrado un importante incremento en su frecuencia, extensión y daño a lo largo del tiempo, y existen evidencias de que esta tendencia continuará si no se actúa en consecuencia (Schwindt, et al., 2020).

El transporte de especies invasoras está a menudo, pero no exclusivamente, asociado con el transporte de actividades comerciales de los buques (por ejemplo, incrustaciones en el casco y/o en el lastre húmedo y seco), convirtiendo a los entornos portuarios en sitios de ingreso para las invasiones biológicas. En la actualidad, el $90 \%$ del comercio global ocurre a través de barcos que, advertida e inadvertidamente, transportan especies acuáticas de una región a otra (Organización Marítima Internacional, 2004).

Las primeras embarcaciones, cuyos cascos eran de madera, utilizaban rocas y arena (lastre seco) para mantener su estabilidad y equilibrio; y entre éstas viajaban de forma involuntaria cientos de pequeños mamíferos, aves, plantas, insectos y semillas. Asimismo, el exterior de los barcos se encontraba colonizado por organismos marinos como algas, moluscos y crustáceos, entre otros. A partir de 1890, con la modernización de la industria naval y la utilización de acero para la construcción de buques, el lastre seco fue reemplazado por tanques especialmente diseñados para contener agua. Con este avance, las embarcaciones comenzaron a ser más veloces y les permitió visitar mayor número de puertos en el mundo (GEF, et al., 2007).

El agua de lastre es esencial para la seguridad y operación eficiente de los buques ya que provee balance, estabilidad, maniobrabilidad y eficacia de propulsión a los 
barcos sin carga (Gollasch, et al., 2007; GEF, et al., 2007). Cuando un buque carga agua de lastre, lo hace junto con los organismos que habitan el lugar, tanto planctónicos como bentónicos y nectónicos, y muy frecuentemente también incorpora sedimentos. Aunque no todos estos organismos sobrevivirán en el tanque de agua de lastre, dado que es un medio hostil con bastante movimiento, falta de alimento y luz; algunos lo hacen, tanto en su forma vegetativa como en forma de huevos, quistes o estadios de resistencia. Esos sobrevivientes, al ser descargados junto al agua de lastre en un puerto diferente al de origen, podrían generar consecuencias desastrosas para el ecosistema local.

Debido a la magnitud e intensidad del impacto generado por las especies exóticas invasoras en todo el mundo, la Organización Marítima Internacional (OMI) ha adoptado diferentes medidas dirigidas al control y manejo del agua de lastre. En 1997, la OMI elaboró y publicó las "Directrices para el control y la gestión del agua de lastre de los buques a fin de reducir al mínimo la transferencia de organismos acuáticos perjudiciales y agentes patógenos" (Organización Marítima Internacional, 1997). Posteriormente, elaboró un instrumento jurídico internacional: el "Convenio internacional para el control y la gestión del agua de lastre y los sedimentos de los buques" (Ballast Water Managment, por sus siglas en inglés, BWM 04) (Organización Marítima Internacional, 2004), que entró en vigencia en setiembre de 2017.

En 1998, la República Argentina se convirtió en el país pionero de Sudamérica en prevenir la introducción de especies exóticas invasoras. A través de su autoridad marítima, la Prefectura Naval Argentina (PNA), se dictó la Ordenanza N 7/98 (DPAM) "Prevención de la contaminación con organismos acuáticos en el lastre de barcos destinados a puertos argentinos en la Cuenca del Plata", basado en Directrices de la OMI (Argentina. Prefectura Naval Argentina, 1998). Esta ordenanza establecía que todos los buques oceánicos con destino a puertos argentinos, que provengan de puertos extranjeros y que lleven agua de lastre a bordo, debían intercambiarla antes de ingresar a las vías fluviales nacionales. Esto se debe a que el intercambio físico de agua de lastre en altamar reduce drásticamente el riesgo de invasión de invertebrados de agua dulce, principalmente debido al choque de salinidad que sufren los organismos que permanecen en los tanques después del intercambio (Bailey, et al., 2011).

Por su parte, la República Oriental del Uruguay, en el año 2006, a través de su autoridad marítima, la Prefectura Nacional Naval (PNN), estableció la Disposición Marítima N¹09 "Directrices para el control y la gestión del agua de lastre de los buques", con objetivos similares a los esperados en el BWM 04 y en la reglamentación de su par rioplatense (Uruguay. Prefectura Nacional Naval, 2006). 
Hacia fines de 2017, Argentina ratificó el Convenio BWM 04 y originó la ordenanza DPAM N 07/2017 "Normas para el control y la gestión del agua de lastre y los sedimentos de los buques, artefactos navales u otras construcciones flotantes" (Argentina. Prefectura Naval Argentina, 2017), donde se exige que todos los barcos que ingresen en aguas jurisdiccionales del país deben realizar la correcta gestión de sus aguas de lastre. Al momento de arribar a puerto, los inspectores realizan controles documentales del Certificado Internacional de Agua de Lastre, el Plan de gestión y sedimentos, y el Libro Registro, donde se asienta la operatoria de gestión de las aguas de lastre. En el caso de que los buques hayan realizado el cambio de sus aguas de lastre en aguas oceánicas, se le realiza un control adicional, midiendo la salinidad. El mismo consiste en la toma de muestras de agua de tanques de lastre, utilizando equipos digitales específicos, y el control de su salinidad (ésta nunca debe exceder los 30 ppm). En caso de incumplimiento, la autoridad marítima local puede aplicar sanciones y prohibir cualquier operación hasta que se realice la descarga en tierra o el intercambio del agua de lastre correcto. Las sanciones se aplican de acuerdo con la gravedad en la violación de la ley y la amenaza potencial para el medio ambiente acuático (Abelando, et al., 2020).

Para cumplir con el BWM 04, los buques deben gestionar su agua de lastre de acuerdo con la norma de cambio de agua de lastre (Regla D-1) o a través de la norma de eficiencia (Regla D-2). Según el calendario para la implantación de la norma de eficiencia (D-2), se obliga a toda la flota de navegación internacional a poseer una planta de tratamiento a más tardar el 8 de septiembre de 2024, definido en la enmienda a la Regla B-3 del BWM 04 (Organización Marítima Internacional, 2018).

Los buques que efectúen el cambio de agua de lastre lo harán a por lo menos 200 millas marinas de la tierra más próxima y en aguas de 200 metros de profundidad como mínimo. De no ser posible, deberá efectuarse a por lo menos 50 millas marinas de la tierra y en aguas de 200 metros de profundidad como mínimo. Este procedimiento debe realizarse con una eficacia del $95 \%$ de cambio volumétrico del agua de lastre. Mientras que los buques que utilicen la Regla D-2, deberán tener a bordo un sistema de tratamiento del agua de lastre aprobado por OMI. Estos sistemas consisten en equipos que utilizan procedimientos mecánicos, físicos y/o químicos que permiten neutralizar o eliminar a los organismos acuáticos perjudiciales y agentes patógenos o reducir su volumen acorde a los niveles establecidos en el convenio. El equipo utilizado debe garantizar que, al llegar a puerto, el buque descargará agua de lastre previamente tratada y con un contenido de menos de 10 organismos viables por metro cúbico, cuyo tamaño mínimo sea igual o superior a 50 micras; y menos de 10 organismos viables por mililitro, cuyo tamaño mínimo sea inferior a 50 micras y superior a 10 micras. Asimismo, las 
cantidades de microbios indicadores no deben exceder determinadas concentraciones: Vibrio cholerae toxicógeno (O1 y O139), menos de 1 unidad formadora de colonias (UFC) por 100 mililitros o menos de 1 UFC por gramo (peso húmedo) de muestras de zooplancton; Escherichia coli, menos de 250 UFC por 100 mililitros, y enterococos intestinales, menos de 100 UFC por 100 mililitros.

El objetivo del presente trabajo es realizar una revisión de las tareas de control en la gestión del agua de lastre en los puertos argentinos situados en la Cuenca del Plata, y verificar si los controles fueron efectivos para evitar el ingreso de nuevas especies invasoras.

\section{MATERIALES Y MÉTODOS}

En la Cuenca del Plata, las especies invasoras más estudiadas y emblemáticas son el mejillón dorado Limnoperna fortunei (Dunker, 1857) y dos especies de almejas, Corbicula fluminea (Müller, 1774) y C. largillierti (Philippi, 1844), que afectan económicamente a las industrias, y a las centrales termoeléctricas e hidroeléctricas. En el caso del mejillón dorado, se requieren operaciones de limpieza muy costosas de equipos utilizados en las centrales hidroeléctricas para mantenerlos libres de esta especie y asegurar su funcionamiento eficiente (Darrigran y Damborenea, 2005; Boltovskoy, et al., 2006). En consecuencia, y hasta no entrar en vigencia el BWM 04, los países afectados debieron tomar una serie de medidas individuales relacionadas con los problemas causados por la introducción de organismos invasores y agentes patógenos a través de los buques.

El trabajo preventivo, como el control en el cumplimiento del BWM 04 que realizan las autoridades marítimas, es fundamental para evitar el ingreso de especies invasoras en la Cuenca del Plata, como así también la investigación científica para poder lograr controles más eficientes, particularmente en la verificación del cumplimiento de la Regla D-2.

En este estudio se analizan datos publicados e inéditos acerca del cumplimiento de la normativa nacional e internacional, análisis de riesgo, tendencias y comparaciones con otros países limítrofes, con especial énfasis en el trabajo realizado en los últimos años por la autoridad marítima argentina.

Desde el año 2011, la PNA realiza inspecciones exhaustivas a través de personal capacitado en los puertos fluviales para verificar el cumplimiento de las regulaciones nacionales. El procedimiento consta de varias etapas:

1. Previo arribo del buque: entre las 24 y 48 horas anteriores al arribo de un buque a puerto, la agencia marítima, como representante del mismo en el país, envía a la PNA la información necesaria para que evalúe si se le permitirá o no el deslastrado 
de sus aguas en el puerto. Los documentos enviados son el Certificado Internacional de Gestión de agua de lastre vigente y el último reporte de cambio o tratamiento de agua de lastre. En el caso de que el buque utilice el método de cambio de agua de lastre (Regla D-1), la información presentada en el reporte permite georreferenciar en el Sistema Guardacostas (software propio PNA) el sitio donde se realizó el cambio del agua y verificar si efectivamente se efectuó acorde a lo establecido en la normativa vigente.

2. Arribo del buque a puerto: personal capacitado de PNA aborda el buque para realizar la toma de muestras de agua de los tanques a deslastrar y verificar su salinidad (Figura 1). Para ello se utiliza un equipo salinómetro marca WTW 315. El agua contenida en los tanques debe tener una salinidad superior a $30 \mathrm{ppm}$. Valores menores indican que existe la posibilidad de que se trate de agua tomada a bordo en un ambiente costero, o que el reemplazo llevado a cabo en altamar fue insuficiente o inefectivo. Si la documentación y la medición de salinidad son correctos se le permite el deslastre en puerto.

3. Luego de la inspección, se transfieren los datos obtenidos a la planilla de Control de Gestión de Agua de Lastre y se envía la información obtenida a la Dirección de Protección Ambiental. Allí se realiza el análisis estadístico de los datos recabados en todos los puertos para generar mejoras en los controles y así reducir al mínimo el riesgo de introducción de organismos acuáticos no deseados.
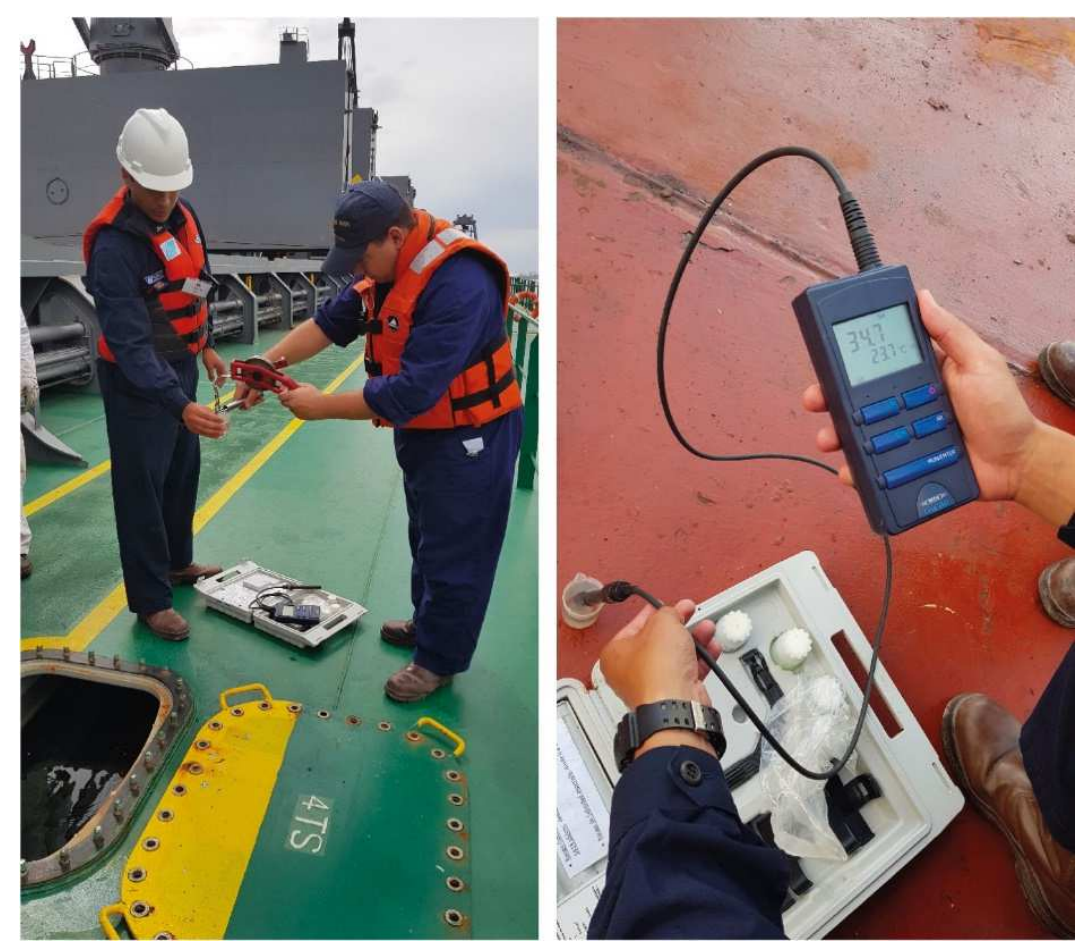
Figura 1. Personal de la PNA realizando medición de salinidad en un tanque de lastre en el puerto de Gral. San Martín-San Lorenzo, provincia de Santa Fe, Argentina.

\section{RESULTADOS Y DISCUSIÓN}

\section{Riesgo de invasión}

Varios autores señalan que la frecuencia e intensidad (o tamaño) de la inoculación son componentes críticos para los casos de riesgo de invasión (Ruiz, et al., 1997; Santagata, et al., 2008; National Research Council, 2011; Castro, et al., 2018). Los puertos colonizados se convierten posteriormente en fuentes de especies invasoras para las áreas vecinas, transportadas por buques que realizan transporte nacional y embarcaciones de recreo, a través del agua de lastre o por bioincrustaciones (Rup, et al., 2010). Además, cuanto mayor sea la similitud ambiental entre el puerto donador y el receptor, mayor será la probabilidad de éxito de la invasión biológica (Carlton, 1985).

La conexión de los principales puertos y terminales fluviales de la Cuenca del Plata con el océano es la vía fluvial más importante de la República Argentina, y resulta vital para el comercio exterior del país (Argentina. Ministerio de Planificación Federal, Inversión Pública y Servicios, 2008). La mayoría de los puertos argentinos se encuentran ubicados sobre el Río Paraná y el Río de la Plata, desde la ciudad de San Lorenzo, en Santa Fe, hasta Ensenada, en Buenos Aires (Figura 2). 


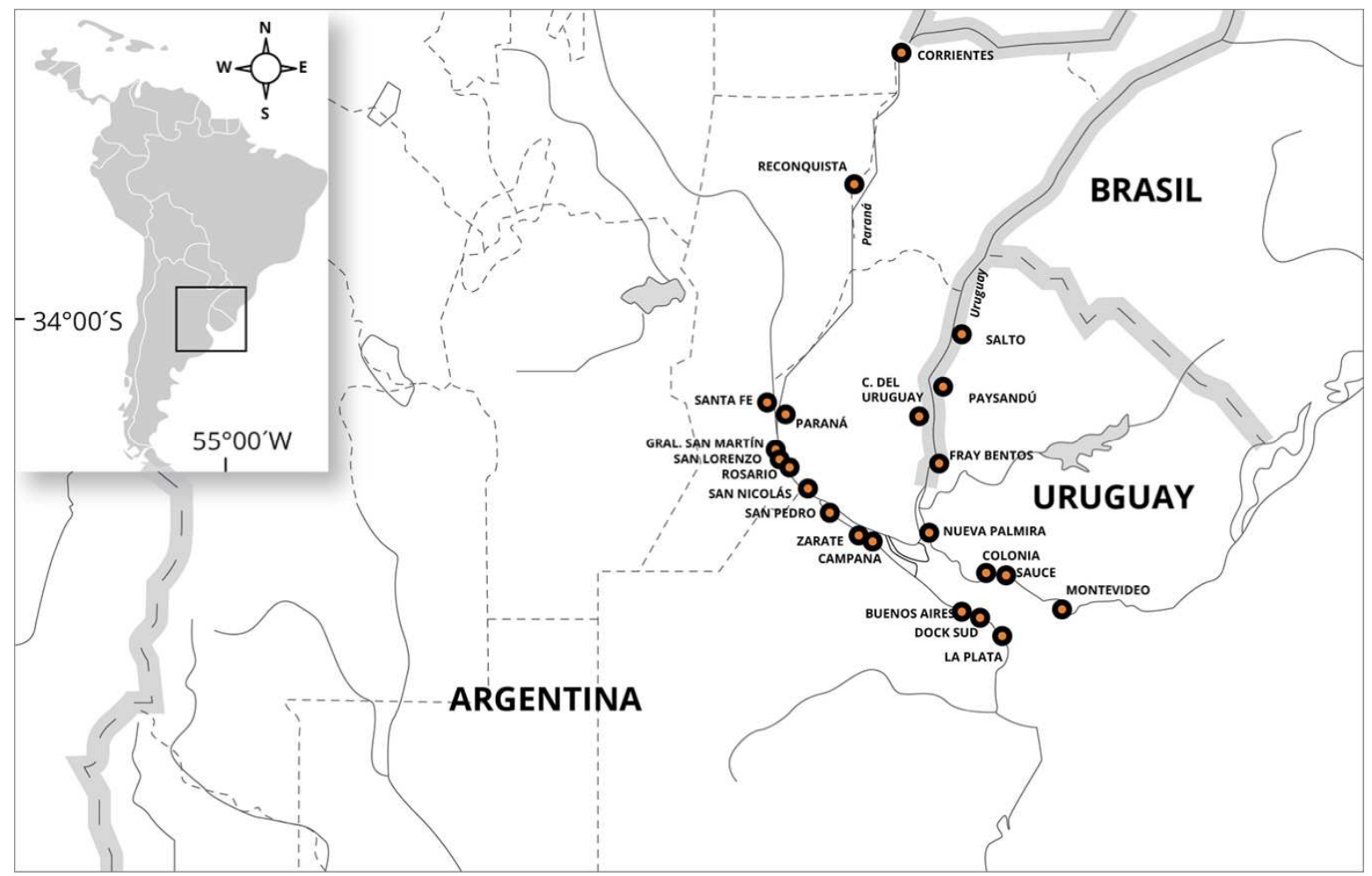

Figura 2. Puertos argentinos y uruguayos más importantes ubicados en la parte inferior de la Cuenca del Plata.

El Ministerio de Agricultura, Ganadería y Pesca realiza el seguimiento de las exportaciones de granos, aceite y subproductos que se exportan en la República Argentina. En la Figura 3 se observa que los puertos con mayor tasa de exportación en la Cuenca del Plata son Gral. San Martín-San Lorenzo y Rosario, ubicados ambos a orillas del Rio Paraná. En el año 2019, las exportaciones en el país estuvieron representadas en un $62.4 \%$ por la venta de cereales y en un $14 \%$ por las semillas y frutos oleaginosos. Mientras que el resto de las exportaciones se vieron representadas por pescados y mariscos (9\%), frutas frescas (5\%), hortalizas y legumbres sin elaborar (3.3\%), tabaco sin elaborar y miel, entre otros (Argentina. Ministerio de Ganadería Agricultura y Pesca, 2020). Estos valores no solo demuestran la importancia de las exportaciones de granos para nuestro país, sino también la intensidad de la inoculación ya que la contrapartida al peso de esa carga se encuentra representada por toneladas de agua de lastre contenida en los buques que llegan a los puertos de la Cuenca del Plata desde diferentes puertos del mundo. 


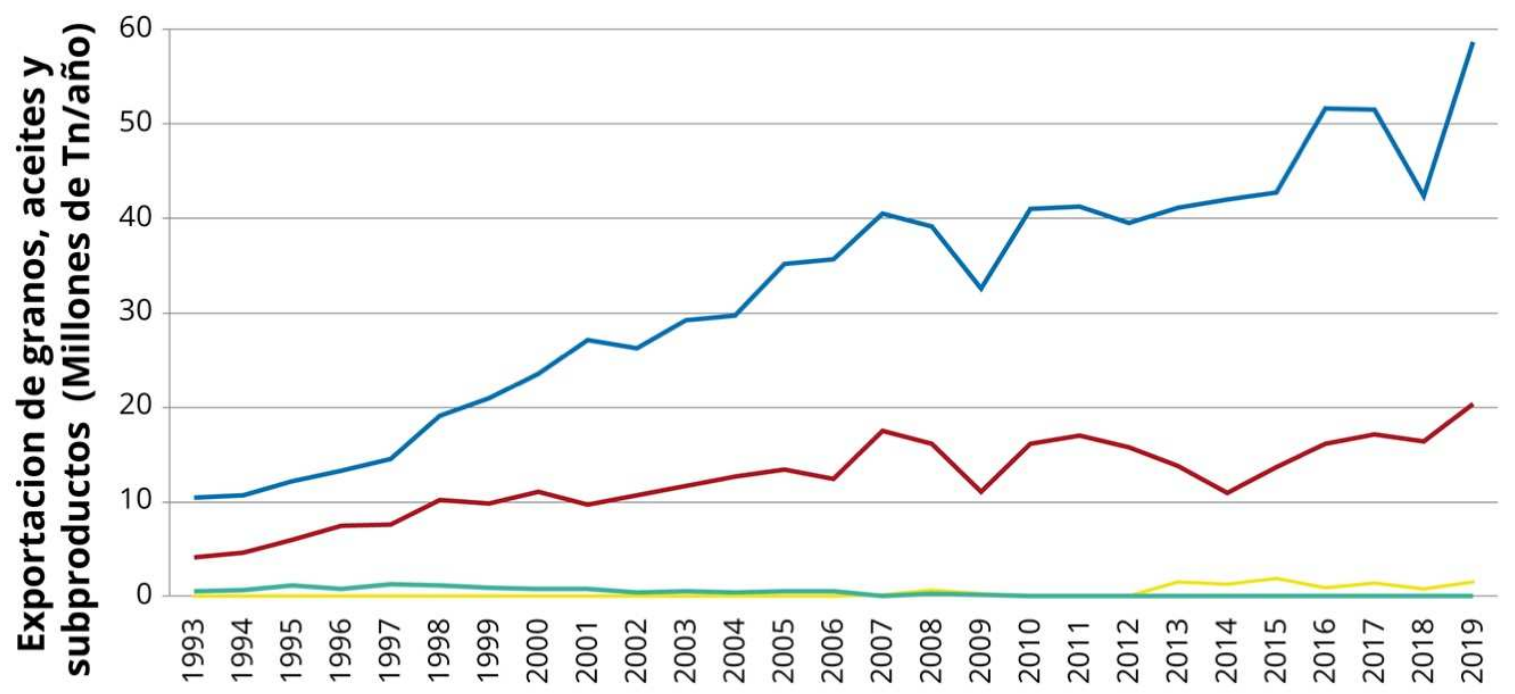

Años

Figura 3. Exportaciones de granos, aceite y subproductos (millones de Tn/año) en los puertos más importantes ubicados sobre el Río Paraná: Gral. San Martín-San Lorenzo (azul), Rosario (rojo), Zarate (amarillo) y el Río de la Plata: Buenos Aires (verde), desde el año 1993 al 2019 (Argentina. Ministerio de Ganadería Agricultura y Pesca, 2020).

Al analizar las inspecciones realizadas desde la entrada en vigencia del BWM 04 (2018-2019) en los principales puertos del país (datos PNA, Figura 4), se observa que los volúmenes se mantienen constantes, especialmente en el puerto de Gral. San Martín-San Lorenzo: al ser el mayor agroexportador de granos y aceites, es uno de los que recibe mayor cantidad de agua de lastre. Los puertos de Zarate, Buenos Aires y La Plata reciben menos cantidad de agua de lastre porque, en general, los buques que allí arriban son de tipo portacontenedores, Ro-Ro o cruceros, los cuales llegan al puerto con carga y vuelven a zarpar con carga, realizando poco movimiento de agua en sus tanques de lastre. 


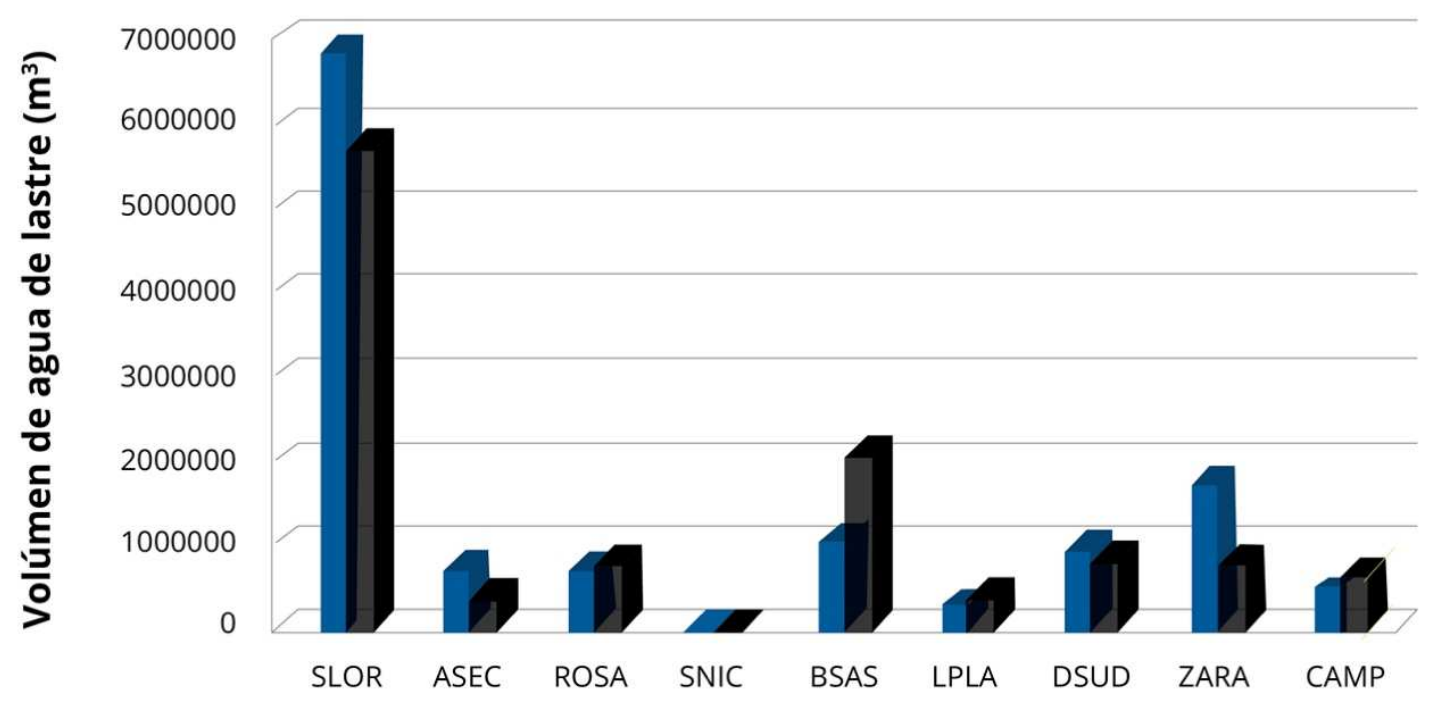

Figura 4. Volumen de agua de lastre descargado en los principales puertos del país durante los años 2018 (azul) y 2019 (negro). Los valores corresponden solo a los volúmenes de inspecciones efectuadas en los años citados. Ref.: SLOR: San Lorenzo, ASEC: Arroyo Seco, ROSA: Rosario, SNIC: San Nicolás, BSAS: Buenos Aires, LPLA: La Plata, DSUD: Dock Sud, ZARA: Zarate, CAMP: Campana.

Abelando y otros (2020) estudiaron las inspecciones realizadas sobre la gestión del agua de lastre a bordo de los buques que arribaron al puerto de Gral. San MartínSan Lorenzo (SLOR), en el año 2017, con el objetivo de evaluar el riesgo de invasiones biológicas en dicho puerto. Ese es el primer estudio exhaustivo realizado en la Cuenca del Plata que combinó el tráfico general de buques, datos ambientales de los puertos globales y las acciones de control realizadas. Las autoras calcularon un Coeficiente de Riesgo Global (CRG), considerando: frecuencia relativa de la cantidad de tanques de lastre de un puerto específico en relación con la cantidad total de tanques descargados (frecuencia de los inóculos); proporción del volumen de agua de lastre de un puerto específico en relación con el volumen total descargado en SLOR (tamaño de los inóculos) y un análisis multivariado, con un coeficiente de distancia euclidiana, de donde se obtuvieron valores de similitud ambiental entre el puerto de recepción de agua de lastre y el último puerto. Obtuvieron que solo el $5 \%$ de los barcos arribados provenían de un puerto fluvial, principalmente de Brasil (Porto Alegre y Santa Clara) y China (Tianjiazhen, Zhongshan, Ma'anshan y Machong). 
Por otra parte, los buques provenientes de puertos con características estuariales, principalmente de Brasil, Singapur y Durban, constituyeron el 37\% del total de las visitas al puerto de SLOR (Figura 5). Ruiz y otros (1997) consideran que los estuarios son los lugares más invadidos, ya que las especies que habitan allí incluyen una amplia gama taxonómica de diversos grupos tróficos (por ejemplo, filtradores, raspadores, colectores, parásitos) y ocupan diferentes hábitats (por ejemplo, sedimentos blandos, sustratos duros, superficie de pantanos y columna de agua). $\mathrm{Si}$ bien desde el punto de vista ambiental los puertos de tipo estuarial muestran una menor similitud con los fluviales, pueden implicar un mayor riesgo de invasión debido a la frecuencia de entrada de barcos con agua de ese origen, y más aún si contienen especies con amplia tolerancia ambiental.

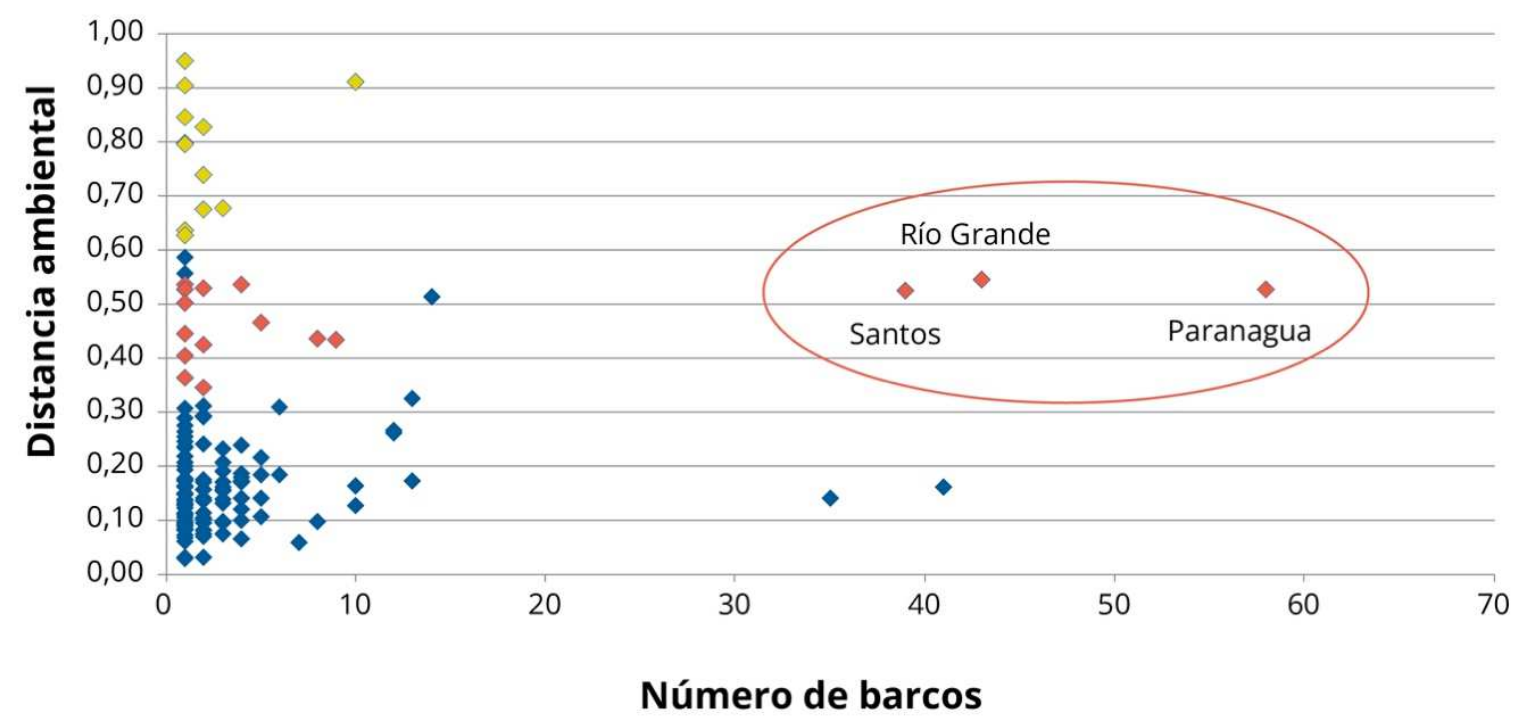

Figura 5. Tráfico de buques y distancia ambiental (calculada en base a la salinidad y temperatura de cada puerto fuente y el Puerto de San Lorenzo). Azul: puertos marinos, rojo: puertos estuariales y amarillo: puertos fluviales. Los puertos marcados con un óvalo son los que aportan la mayor cantidad de agua de lastre. Fuente: (Abelando, et al., 2020).

Al momento del control, los procedimientos de muestreo de agua de lastre pueden ser uniformes o selectivos para los buques que arriban a un determinado puerto. En los muestreos uniformes, todos los barcos son sometidos a los mismos procedimientos estandarizados, lo que los hace costosos y técnicamente poco prácticos (Ta-Kang, et al., 2014). Para evaluar el riesgo de invasiones en los puertos, Abelando y otros (2020) proponen conocer cuáles son los últimos puertos de parada 
y las rutas marítimas de las embarcaciones que arriban a cada puerto del país. Con un control selectivo de las embarcaciones, considerando el origen del agua de lastre y la frecuencia de arribo a cada puerto, las autoridades marítimas podrían optimizar sus recursos humanos y económicos. Un análisis de riesgo similar podría aplicarse en el control Norma D-2, incluyendo algún coeficiente específico.

\section{Efectividad en los controles}

\section{A) Cambio del agua de lastre (Regla D-1)}

En cuanto al control en el cumplimiento de las ordenanzas nacionales en los últimos años y la normativa internacional (BWM 04), se observó que el grado de cumplimiento por parte de la flota internacional ha mejorado sustancialmente en algunos países de Sudamérica (Castro, et al., 2017, Abelando, et al., 2020).

En Argentina existían dos antecedentes dirigidos a estudiar el movimiento portuario asociado con el agua del lastre de buques, uno principalmente en puertos marítimos de la Patagonia (Boltovskoy, et al., 2011) y otro en el puerto de Buenos Aires (Ávila Velandia, 2016). En el primer estudio, realizado en el puerto fluvial de Dock Sud (Buenos Aires, Argentina) y en cuatro puertos marítimos durante el periodo 2007 y 2008, se observó que en la mayoría de los buques las tripulaciones estaban familiarizadas con las regulaciones de la OMI. Sin embargo, en más del $70 \%$ de los buques inspeccionados, las regulaciones argentinas eran desconocidas y en el $60 \%$ era imposible establecer la fuente del agua de lastre. Ávila Velandia (2016) obtuvo resultados similares entre 2010 y 2011 para el puerto de Buenos Aires, donde más de la mitad de las inspecciones contenían errores, omisiones o inconsistencias en las planillas presentadas.

A diferencia de las observaciones hechas por estos investigadores, previas a la entrada en vigencia del BWM 04; Abelando y otros (2020) observaron que la documentación presentada por los buques en el puerto de San Lorenzo era correcta, así como también la gestión del agua de lastre a bordo. Esta mejora sustancial en el cumplimiento podría deberse, en parte, a la entrada en vigencia del citado convenio; y también a que, durante la última década, la Prefectura Naval Argentina aumentó la cantidad de inspecciones a bordo e incorporó una serie de cursos de capacitación para su personal, lo que permitió mayor control y eficiencia en las mismas.

En otros países de la región como Brasil, Pereira y otros (2018) realizaron un estudio entre los años 2012 y 2013 en el Puerto de Santana, ubicado en la región amazónica, donde observaron que la efectividad del cambio del agua de lastre mostró deficiencias considerables, como por ejemplo fallas en la información sobre el origen y los volúmenes de intercambio de agua de lastre. Castro y otros (2017) 
realizaron un trabajo similar en diferentes puertos de Brasil, entre los años 2005 y 2015, y luego de 10 años de esfuerzo en los controles de la autoridad marítima, se evidenciaron altos niveles de cumplimiento con las regulaciones de gestión del agua de lastre. Sin embargo, también mostraron discrepancias en el régimen de inspección entre puertos a lo largo de la costa. Los autores consideraron que esta disminución en el incumplimiento probablemente refleja un aumento en el conocimiento de la norma brasilera y un mayor compromiso de la industria naviera para minimizar la propagación de especies invasoras.

Las regulaciones nacionales que exigen el intercambio de agua de lastre en medio del océano, utilizando el cambio osmótico para eliminar a las especies costeras, se ha implementado en muchos lugares del mundo desde principios de la década de 1990. Si bien existe evidencia de que el intercambio de agua de lastre ha reducido efectivamente las introducciones en los Grandes Lagos (Canadá y Estados Unidos), donde se observó un alto nivel de aplicación, aún persiste una gran incertidumbre sobre el grado de protección que este método ofrece a las zonas marinas costeras (Bailey, et al., 2020).

En la Cuenca del Plata, donde se encuentran los puertos comerciales de mayor actividad (Buenos Aires, La Plata y Montevideo), además de Limnoperna fortunei (Dunker, 1857), registrada por primera vez en 1991 (Pastorino, et al., 1993), y las almejas Corbicula fluminea (Müller, 1774) y C. largillierti (Philippi, 1844) durante 1970 (Ituarte, 1981), se han establecido otras especies. Entre ellas, el gasterópodo Rapana venosa (Valenciennes, 1846) en 1998 (Scarabino, et al., 1999 y Pastorino, et al., 2000), la medusa Blackfordia virginica (Mayer, 1910) en el año 2000 (Genzano, et al., 2006) y Manayunkia speciosa (Leidy, 1858) registrada en 2007, presente solo en el Río Uruguay (Armendariz, et al., 2011). La mayoría de estas especies fueron citadas por primera vez entre los años 1979 y 2007, siendo los buques el vector primario de ingreso (agua de lastre y/o bioincrustaciones), no reportándose nuevas especies invasoras en la última década.

Una situación diferente fue la observada por Schwindt y otros (2020), en el sudoeste del Océano Atlántico, al realizar una revisión de las especies invasoras, tomando como base el trabajo de Orensanz y otros (2002), primer estudio completo de organismos marinos exóticos, donde databa la presencia de 31 especies introducidas y 46 criptogénicas. Esta nueva revisión completa ha demostrado que durante las últimas dos décadas el número de introducciones supera las 120 especies, cuatro veces el número informado anteriormente.

Del análisis de la efectividad de los controles hasta el presente, el cual se ha hecho principalmente a través de la verificación de la Regla D-1 en la Cuenca del Plata, se podría inferir que estos han sido efectivos para evitar el ingreso de nuevas especies invasoras en el transcurso de la última década; contrariamente a lo ocurrido en el 
Mar Argentino, donde la medición de salinidad no es útil para discernir el origen del agua de lastre. Se necesitan más investigaciones para verificar si existe algún tipo de relación entre la aparición de nuevas especies, el cumplimiento de la normativa vigente y la frecuencia e intensidad del tráfico marítimo.

B) Eficiencia para el tratamiento del agua de lastre (Regla D-2)

Con la entrada en vigencia del BWM 04, los buques tienen un plazo determinado para instalar a bordo sistemas de tratamiento de agua de lastre y dejar de utilizar el cambio de agua de lastre como medida de gestión. Acorde a la última enmienda realizada al BWM 04 (Organización Marítima Internacional, 2018), referente al calendario para la implantación de la norma de eficiencia (Regla D-2), los buques deben instalar una planta de tratamiento según la fecha de renovación del certificado internacional de prevención de la contaminación por hidrocarburos, conocido como Convenio MARPOL (Organización Marítima Internacional, 1973).

Esta norma de eficacia es el resultado de prolongadas negociaciones entre los Estados Miembros de la OMI, y representa un compromiso cuya finalidad es promover el desarrollo de opciones más seguras y eficaces de gestión del agua de lastre. El desarrollo y la aprobación de los sistemas de tratamiento requiere de una serie de pruebas de desempeño teniendo en cuenta diferentes variables como: tipos de buques existentes, rutas de navegación, condiciones climáticas, posición geográfica, uso o no de sustancias activas y legislación local e internacional (Organización Marítima Internacional, 2004).

En la actualidad, existen en el mercado numerosos equipos de tratamiento aprobados por la OMI e instalados a bordo de las embarcaciones. Del análisis de las inspecciones efectuadas desde la entrada en vigencia del convenio en puertos argentinos, se observó un incremento en la cantidad de buques que poseen estos sistemas (Figura 6). Esto es un buen indicio del compromiso de los países para cumplir con lo establecido en el convenio y contribuir a la preservación de la biodiversidad mundial. 


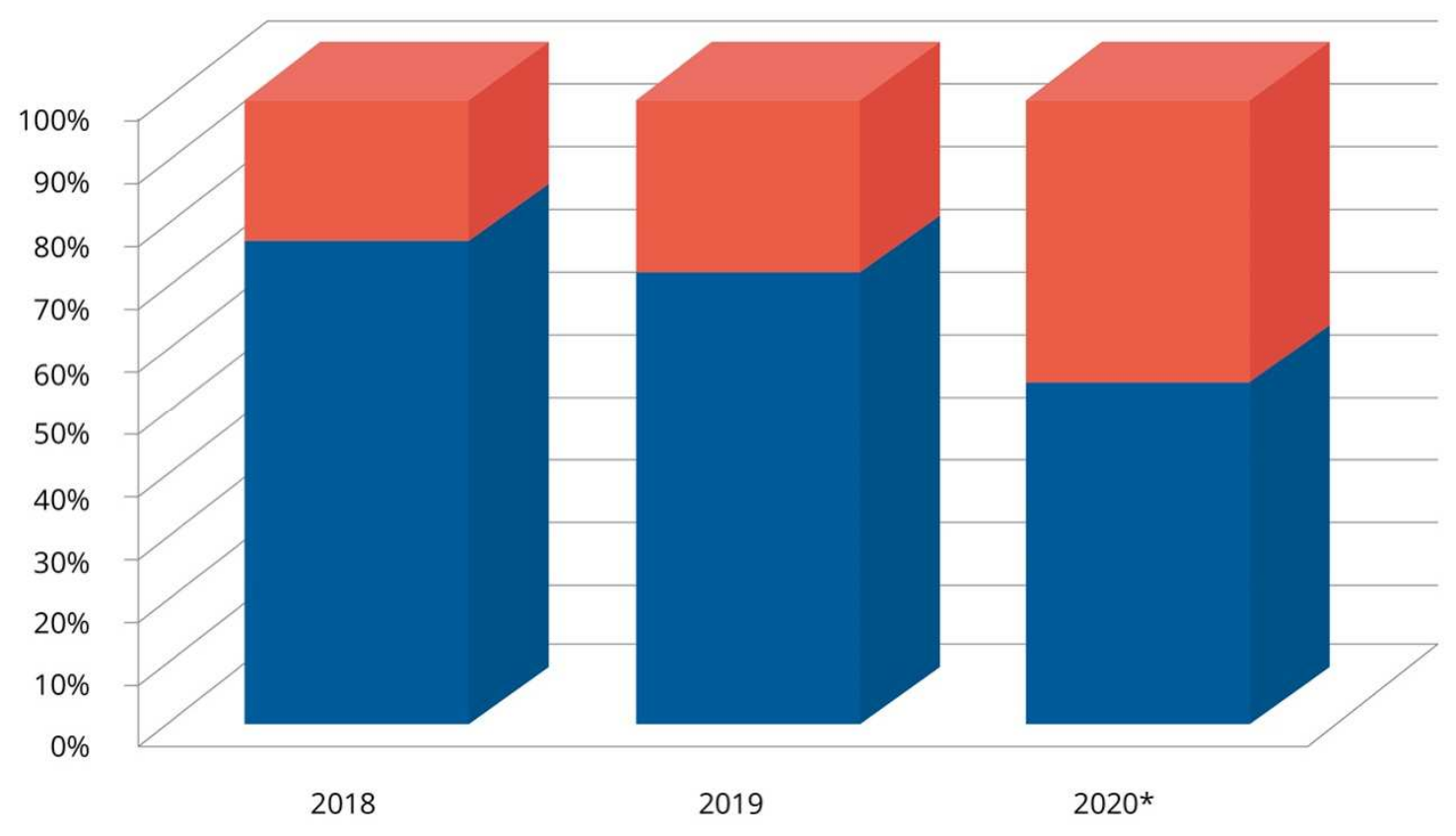

Figura 6. Porcentaje de métodos de gestión del agua de lastre registrado en las inspecciones realizadas en puertos argentinos de la Cuenca del Plata durante los años 2018, 2019 y 2020* (de enero a mayo). Ref.: azul: D-1 y rojo: D-2.

El tratamiento del agua de lastre antes de ser vertida en un puerto provee una mejora sustancial ya que disminuiría la cantidad de potenciales especies invasoras. Sin embargo, hay algunas cuestiones aun no resueltas al respecto, las cuales se encuentran en etapa de estudio y reglamentación.

Una de ellas se basa en una cuestión práctica al momento de realizar una inspección. Como ya se mencionó más arriba, la norma de eficacia se basa en el número de microorganismos y patógenos presentes en el agua de lastre. Esto hace que la verificación del cumplimiento o incumplimiento de esta norma presente algunos inconvenientes como: a) la obtención de una muestra representativa, ya que no todos los tanques tienen el mismo volumen, y los microorganismos presentes poseen tamaño y comportamiento diferentes dentro del tanque; b) el análisis de la presencia de los organismos planctónicos requiere microscopios y personal capacitado en cada puerto, y en el caso de patógenos la infraestructura adecuada para su análisis; c) los métodos de medición de salinidad utilizados para comprobar la Regla D-1 ya no son válidos y d) no generar demoras innecesarias en el buque, siendo que algunos de los análisis requieren un plazo de 24 o 48 horas para la obtención del resultado. 
A pesar de los esfuerzos de los países miembros del Comité de Protección del Medio Ambiente de OMI (MEPC, por sus siglas en inglés), quienes han generado una serie de directrices como las "Orientaciones sobre el muestreo y análisis del agua de lastre" (Organización Marítima Internacional, 2015) y las permanentes enmiendas a fin de perfeccionar las directrices para facilitar las tareas y mejorar sus resultados, aun no se ha logrado el consenso en cuanto a un único método para comprobar la efectividad de la Regla D-2.

Otro punto a considerar guarda relación con que no todos los sistemas de tratamiento garantizan el $100 \%$ de eficiencia, es decir, ninguno de ellos es capaz de erradicar por completo especies exóticas o patógenas del agua de lastre. Varios estudios han señalado problemas con la eficiencia de estos sistemas (Mesbahi, 2004; Cohen y Dobbs, 2015; Paolucci, et al., 2015; Lin, et al., 2020), así como también se han observado problemas de operación con los equipos tales como taponamiento de filtros, falta de biocidas en los puertos para su adquisición, excesiva turbidez, toxicidad, corrosión y falta de compromiso de la tripulación en la operación de los equipos, entre otros (Bakalar, 2016; Pereira y Brinati, 2018).

En la actualidad, la mayoría de los países de Sudamérica no cuentan con la capacidad operativa o técnica de controlar todos los buques que entran a sus puertos o aguas territoriales con sistemas de tratamiento a bordo, por los motivos detallados anteriormente. El problema aún no está totalmente resuelto para la comunidad marítima internacional, y además presenta un gran desafío futuro para los países al momento de realizar los controles en los puertos.

\section{CONCLUSIONES}

La Cuenca del Plata es un gran receptor de agua de lastre proveniente de diferentes puertos del mundo. Por ello, toda medida tendiente a evitar consecuencias negativas en el ambiente, la salud y la economía de una región es necesaria, siendo la prevención reconocida globalmente como la opción más efectiva y deseable para evitar la introducción de nuevas especies y su dispersión en el medio acuático.

Luego de una década de trabajo, en la República Argentina fueron varios los factores que contribuyeron para minimizar el ingreso de especies exóticas invasoras en la Cuenca del Plata, donde desde el año 2011 no se reportaron nuevas invasiones, a diferencia de lo ocurrido en el Mar Argentino. Entre ellos, la efectividad en el control del cumplimiento de las reglamentaciones nacionales e internacionales, el mayor grado de compromiso de la flota mercante y el aumento de la capacidad técnica del personal de la PNA. 
Asimismo, se debe continuar con los esfuerzos y aumentar las investigaciones sobre especies exóticas invasoras en los puertos, como la construcción de una línea de base portuaria y monitoreos frecuentes, la capacitación continua del personal perteneciente a la autoridad marítima, mantener contacto fluido con diferentes actores del ambiente marítimo y portuario, así como también lograr mayor eficiencia en los controles portuarios a través de la optimización de los recursos. En un futuro cercano, también sería importante comenzar a incorporar información relativa a la fuente de origen (análisis de riesgo), a los formularios de agua de lastre y al diseño de sistemas de alerta temprana en los puertos. Todas estas acciones beneficiarán a los países de la región, particularmente en el control del cumplimiento de la Regla D-2, donde las dificultades técnicas y operativas no permiten el control de la totalidad de buques arribados a puertos.

La gestión de las invasiones debe abordarse desde un punto de vista holístico. La cooperación regional es de crucial importancia, en especial en regiones geográficas donde los ecosistemas sobrepasan las fronteras políticas, como lo es la Cuenca del Plata, conformada por cuatro países (Argentina, Brasil, Paraguay y Uruguay). La participación de las autoridades nacionales, tanto marítimas como ambientales, da como resultado asociaciones que facilitan el entendimiento y los enfoques comunes, al igual que el intercambio de información, experiencias, conocimientos científicos y compromisos. Sobre todo, cuando dos de los países (Argentina y Brasil) ya han ratificado el Convenio de agua de lastre y han logrado una experiencia interesante en su implantación.

Otro de los desafíos a futuro es el control de bioincrustaciones en los cascos y zonas "nicho" de los buques (ranuras y oquedades, cajones de toma de mar, mecha y bisagra del timón, guardacabos, juntas de la bocina y ejes portahélices, cadena del ancla, entre otros). Al igual que ocurre con el agua de lastre, los grandes puertos actuarían como regiones receptoras primarias de las especies invasoras que llegan desde una región distante. Una vez establecidas allí, procesos naturales (natación activa, transporte pasivo de larvas o deriva) y mecanismos de dispersión mediados por el hombre (embarcaciones de recreo, acuicultura, pesca y restauración) pueden facilitar su propagación secundaria hacia otras localidades cercanas, logrando así una mayor expansión en su rango de distribución (Occhipinti-Ambrogi, 2007; Floerl, et al., 2005; Ferrario, et al., 2017, Bailey, et al., 2020).

Dada la importancia de esta problemática, la OMI, en su resolución MEPC.207(62) "Directrices de 2011 para el control y la gestión de la contaminación biológica de los buques a los efectos de reducir al mínimo la transferencia de especies acuáticas invasivas" (Organización Marítima Internacional, 2011), recomienda la remoción de bioincrustaciones durante inspecciones periódicas, pero resalta la necesidad -antes de proceder- de evaluar el riesgo para el medio ambiente y los recursos, ya sea por 
la liberación de organismos como por la liberación de tóxicos procedentes de los residuos de las pinturas antiincrustantes.

Por todo lo expuesto, los Programas de Detección Temprana a nivel nacional y regional serán la clave para evitar la expansión de las especies invasoras con un mínimo costo económico y permitirán generar una base de datos de las especies presentes en los puertos para poder detectar las regiones más sensibles, y así escoger las medidas de manejo más adecuadas. Estos programas son útiles tanto para el control del agua de lastre como para el estudio de las bioincrustaciones en los buques.

\section{AGRADECIMIENTOS}

Un agradecimiento especial a la jefatura de la Dirección de Protección Ambiental de la Prefectura Naval Argentina por brindar apoyo para la investigación sobre las invasiones biológicas. A la Dra. Jessica Chiarandini Fiore, la Lic. Magalí Bobinac y la Lic. Laura Abelando por sus sugerencias y comentarios críticos para mejorar este trabajo.

\section{REFERENCIAS}

Abelando, M., Bobinac, M. y Chiarandini Fiore, J., 2020. Assessment of the efficiency of controls to prevent biologic invasions at the San Lorenzo Port, Argentina. En: Environmental Monitoring and Assessment, 192, pp.420. DOI: https://doi.org/10.1007/s10661-020-08359-2

Argentina. Ministerio de Ganadería Agricultura y Pesca, 2020. Exportaciones [En línea]. Buenos Aires: MAGYP. [Consulta: 18 de agosto de 2020]. Disponible en:

https://www.agroindustria.gob.ar/sitio/areas/ss_mercados_agropecuarios/expo rtaciones/

Argentina. Ministerio de Planificación Federal, Inversión Pública y Servicios, 2008.

Plan maestro y director del Sistema de Navegación Troncal. Visión estratégica y bases para su formulación en el Río Paraná [En línea]. Buenos Aires:

Subsecretaría de Puertos y Vías Navegables, Secretaría de Transporte, Ministerio de Planificación Federal. [Consulta: 18 de agosto de 2020]. Disponible en: https://www.estudioayd.com.ar/data/PresentacionPLANyLIBRO.pdf 
Argentina. Prefectura Naval Argentina, 1998. Ordenanza DPAM No 7/98.

Prevención de la contaminación con organismos acuáticos en el lastre de barcos destinados a puertos argentinos en la Cuenca del Plata. Buenos Aires: PNA. (Derogada).

Argentina. Prefectura Naval Argentina, 2017. Ordenanza DPAM No. 07/17. Normas para el control y la gestión del agua de lastre y los sedimentos de los buques, artefactos navales $u$ otras construcciones flotantes [En línea]. Buenos Aires: PNA. [Consulta: 19 de octubre de 2021]. Disponible en: https://www.argentina.gob.ar/prefecturanaval/reglamentacion/ordenanzas

Armendariz, L., Paola, A. y Rodrigues Capítulo, A. 2011. Manayunkia speciosa Leidy (Polychaeta: Sabellidae): introduction of this nonindigenous species in the Neotropical Region (Uruguay river, South America). En: Biological Invasions, 13, pp.281-284. DOI: https://doi.org/10.1007/s10530-010-9838-0

Ávila Velandia, M.F., 2016. Evaluación del riesgo de introducción de especies invasoras en agua de lastre en buques que operan en el puerto de Buenos Aires. Buenos Aires: Universidad de Buenos Aires. (Tesis de Maestría).

Bailey, S., Deneau, M., Jean, L., Wiley, C., Leung, B. y MacIsaac H., 2011. Evaluating efficacy of an environmental policy to prevent biological invasions. En: Environmental Science and Technology, 45(7), pp.2554-2561. DOI: https://doi.org/10.1021/es102655j

Bailey, S., Brown, L., Campbell, M., Canning-Clode, J., Carlton, J., Castro, N,. Chainho, P., Chan, F., Creed, J., Curd, A. y Darling, J.. 2020. Trends in the detection of aquatic non-indigenous species across global marine, estuarine and freshwater ecosystems: A 50-year perspective. En: Biodiversity research, 26(12), pp17801797. DOI: https://doi.org/10.1111/ddi.13167

Bakalar, G., 2016. Comparisions of interdisciplinary ballast water treatment systems and operational experiences from ships. En: SpringerPlus, 5, 240. DOI: https://doi.org/10.1186/s40064-016-1916-z

Boltovskoy, D., Correa, N., Cataldo, D. y Sylvester, F., 2006. Dispersion and ecological impact of the invasive freshwater bivalve Limnoperna fortunei in the Río de la Plata watershed and beyond. En: Biological Invasions, 8(4), pp.947-963. DOI: https://doi.org/10.1186/s40064-016-1916-z 
Boltovskoy, D., Almada, P. y Correa, N., 2011. Biological invasions: assessment of threat from ballast-water discharge in Patagonian (Argentina) ports. En: Environmental Science \& Policy, 14(5), pp.578-583. DOI:

https://10.1016/j.envsci.2011.03.007

Carlton, J.T., 1985. Transoceanics and interoceanics dispersal of coastal marine organisms: the biology of ballast water. En: Oceanography and Marine Biology, 23, pp.313-374.

Carlton, J.T., 1996. Pattern, process, and prediction in marine invasion ecology. En: Biology Conservation, 78, pp.97-106. DOI: https://doi.org/10.1016/0006$\underline{3207(96) 00020-1}$

Castro, M., Hall-Spencer, J., Fonseca Poggian, C. y Filemand, T., 2017. Ten years of Brazilian ballast water management. En: Journal of Sea Research, 133, pp.36-42. DOI: https://doi.org/10.1016/j.seares.2017.02.003

Castro, M., Vance, T., Yunnie, A., Fileman, W. y Hall-Spencer, J., 2018. Low salinity as a biosecurity tool for minimizing biofouling on ship sea chests. En: Ocean Science Discussions, 14, pp.661-667. DOI: https://doi.org/10.5194/os-2018-35

Cohen, A. y Dobbs, F., 2015. Failure of the public health testing program for ballast water treatment systems. En: Marine Pollution Bulletin, 9, pp.29-34. DOI: https://doi.org/10.1016/j.marpolbul.2014.12.031

Darrigran, G. y Damborenea, C., 2005. A South American bioinvasion case history: Limnoperna fortunei (Dunker, 1857), the golden mussel. En: American Malacological. Bulletin, 20, pp.105-112.

Floerl, O., Inglis, G.J. y Hayden, B.J., 2005. A risk-based predictive tool to prevent accidental introductions of nonindigenous marine species. En: Environmental Management, 35(6), pp.765-778. DOI: https://doi.org/10.1007/s00267-004$\underline{0193-8}$

Ferrario, J., Caronni, S., Occhipinti-Ambrogi, A. y Marchini, A., 2017. Role of commercial harbours and recreational marinas in the spread of nonindigenous fouling species. En: Biofouling, 33(8), pp.651-660. DOI: https://doi.org/10.1080/08927014.2017.1351958 
GEF, PNUD e IMO, 2007. Building Partnerships to assist developing countries to reduce the transfer of harmful aquatic organisms in ship's ballast water. [En línea]. Londres: Globallast Partnerships [Consulta: 19 de octubre de 2021]. Disponible en: http://archive.iwlearn.net/globallast.imo.org/

Genzano, G., Mianzan, H., Acha, E.M. y Gaitan, E., 2006. First record of the invasive medusa Blackfordia virginica (Hydrozoa: Leptomedusae) in the Río de La Plata, Argentina-Uruguay. En: Revista Chilena de Historia Natural, 79, pp.257-261.

Gollasch, S., Matej, D., Voigt, M., Dragsund, E., Hewitt, C. y Fukuyo, Y., 2007. Critical review of the IMO International Convention of the management of ships ballast water and sediments. En: Hamful algae, 6, pp.585-600. DOI: https://doi.org/10.1016/j.hal.2006.12.009

Ituarte, C.F., 1981. Primera noticia acerca de la introducción de pelecípodos asiáticos en el área rioplatense (Mollusca Corbiculidae). En: Neotrópica, 27, pp.79-82.

Lin, Y., Zhan, A., Hernandez, M., Paolucci, E., MacIsaac, H. y Briski, E., 2020. Can chlorination of ballast water reduce biological invasions? En: Journal of Applied Ecology, 57, pp.331-343. DOI: https://doi.org/10.1111/1365$\underline{2664.13528}$

Mesbahi, E., 2004. Latest results from testing seven different technologies under the EU MARTOB project- Where do we stand now? En: Matheickal, J., Raaymakers, S., eds. Second International Symposium on Ballast Water Treatment. Londres: IMO, pp.210-230.

National Research Council, 2011. Assessing the relationship between propagule pressure and invasion risk in ballast water. Washington, DC: The National Academies Press. DOI: https://doi.org/10.17226/13184.

Occhipinti-Ambrogi, A., 2007. Global change and marine communities: alien species and climate change. En: Marine Pollution Bulletin, 55(7), pp.342-352. DOI: https://doi.org/10.1016/j.marpolbul.2006.11.014

Organización Marítima Internacional, 1973. MARPOL. Convenio internacional para prevenir la contaminación por los buques, modificado por el Protocolo de 1978 y por el Protocolo de 1997 MARPOL [En línea]. Londres: OMI. [Consulta: 19 de octubre de 2021]. Disponible en: https://www.imo.org/es/About/Conventions/Pages/International-Conventionfor-the-Prevention-of-Pollution-from-Ships-(MARPOL).aspx. 
Organización Marítima Internacional, 1997. Resolución A.868(20). Directrices para el control y la gestión del agua de lastre de los buques a fin de reducir al mínimo la transferencia de organismos acuáticos perjudiciales y agentes patógenos. Londres: OMI.

Organización Marítima Internacional, 2004. International convention for the control and management of ship's ballast water and sediments. Londres: OMI. ISBN 978-92-801-033-4

Organización Marítima Internacional, 2011. MEPC.207(62) Add1. Guidelines for the control and management of ships' biofouling to minimize the transfer of invasive aquatic species. Londres: OMI.

Organización Marítima Internacional, 2015. BWM.2/Circ.42/Rev.1. Guidance on ballast water sampling and analysis for trial use in accordance with the BWM Convention and Guidelines (G2). Londres: OMI

Organización Marítima Internacional, 2018. MEPC.297(72) Enmiendas al Convenio internacional para el control y la gestión del agua de lastre y los sedimentos de los buques, 2004. Enmiendas a la regla B-3. Calendario para la implantación de la gestión del agua de lastre de los buques. Londres: OMI

Orensanz, J.M., Schwindt, E., Pastorino, G., Bortolus, A., Casas, G., Darrigran, G., Elías, R., López Gappa, J.J., Obenat, S., Pascual, M., Penchaszadeh, P., Piriz, M.L., Scarabino, F., Spivak, E.D. y Vallarino, E.A., 2002. No longer a pristine confine of the world ocean-a survey of exotic marine species in the Southwestern Atlantic. En: Biological Invasions, 4, pp.115-143. DOI: https://doi.org/10.1023/A:1020596916153

Paolucci, E., Hernandez, M., Potapov, A., Lewis, M. y MacIsaac, H., 2015. Hybrid system increases efficiency of ballast water treatment. En: Journal of Applied Ecology, 52(2), pp. 348-357. DOI: https://doi.org/10.1111/1365-2664.12397

Pastorino, G., Darrigran, G., Martin, S. y Lunaschi, L., 1993. Limnoperna fortunei (Dunker, 1857) (Mytilidae), nuevo bivalvo invasor en aguas del Río de la Plata. En: Neotrópica, 39, pp.101-102.

Pastorino, G., Penchaszadeh, P.E., Schejter, L. y Bremec, C., 2000. Rapana venosa (Valenciennes, 1846) (Mollusca: Muricidae). A new gastropod in South Atlantic waters. En: Journal of Shellfish Research, 19, pp.897-899. 
Pereira, J., Pereira, N. y da Cunha, A., 2018. Monitoramento da qualidade da água de lastro como suporte à gestão no Porto de Santana-AP - Amazônia Estuarina/Brasil. Cap. 8. En: Pereira, Newton Narciso, ed. Água de lastro: gestão e controle. São Paulo: Blucher. pp.171-197.

Pereira, N. y Brinati, H. 2018. Alternativas de tratamentos para água de lastro a bordo dos navios. Cap. 3. En: Pereira, Newton Narciso, ed. Água de lastro: gestão e controle. São Paulo: Blucher. pp.43-88.

Pimentel, D., Rodolfo, Z. y Morrison, D., 2005. Update on the environmental and economic costs associated with alien invasive species in the United States. En: Ecological Economics, 52(3), pp.273-288.

Ruiz, G., Carlton, J., Grosholz, E. y Hines, A., 1997. Global invasions of marine and estuarine habitants by non-indigenous species: mechanism, extent and consequences. En: American Zoologist, 37(6), pp.621-632.

Rup, M.P., Bailey, S.A., Wiley, C.J., Minton, M.S., Miller, A.W., Ruiz, G.M. y MacIsaac, H.J., 2010. Domestic ballast operations on the Great Lakes: potential importance of Lakers as a vector for introduction and spread of nonindigenous species. En: Canadian Journal of Fisheries and Aquatic Sciences, 67(2), pp.256-268. DOI: https://doi.org/10.1139/F09-180

Santagata, S., Gasiunaite, Z., Verling, E., Cordell, J., Eason, K., Cohen, J., Bacela, K., Quilez-Badia, G., Johengen, T., Reid. D. y Ruiz, G., 2008. Effect of osmotic shock as a management strategy to reduce transfers of nonindigenous species among low-salinity ports by ships. En: Aquatic Invasions, 3, pp.61-76. DOI: https://doi.org/10.3391/ai.2008.3.1.10

Scarabino, F., Menafra, R. y Etchegaray, P., 1999. Presencia de Rapana venosa (Valenciennes, 1846) (Gastropoda: Muricidae) en el Río de la Plata. En: Boletín de la Sociedad Zoológica del Uruguay, 11, 40.

Schwindt, E., Battini, N., Giachetti, C. y Bortolus, A., 2018. Especies exóticas marinocosteras de Argentina / Marine-coastal exotic species of Argentina. Editor: Alejandro Bortolus y Evangelina Schiwndt. Puerto Madryn, Argentina. ISBN: 978-987-42-9642-9

Schwindt, E., Carlton, J.T., Orensanz, J.M., Scarabino, F. y Bortolus, A., 2020. Past and future of the marine bioinvasions along the Southwestern Atlantic. En: Aquatic Invasions 15(1), pp.11-29. DOI: https://doi.org/10.3391/ai.2020.15.1.02 
Ta-Kang, L., Chang, Ch-H. y LiChou, M., 2014. Management strategies to prevent the introduction of non-indigenous aquatic species in response to the Ballast Water Convention in Taiwan. En: Marine Policy, 44, pp.187-195. DOI: https://doi.org/10.1016/j.marpol.2013.08.023

Uruguay. Prefectura Nacional Naval, 2006. Disposición Marítima Nº 109 Directrices para el control y la gestión del agua de lastre de los buques. República Oriental del Uruguay [En línea]. Montevideo: PNN. [Consulta: 18 de agosto de 2020]. Disponible en:

http://www.armada.mil.uy/ContenidosPDFs/Prena/Dirme/disposiciones_mariti mas/disposicion_maritima_109.pdf 